three cases of spontaneous haemorrhage in the spinal canal, and the history of this condition, as it appears in the literature, was presented. In none of the cases did the haemorrhage result from an angioma; in two cases the bleeding was subdural and in one case extradural. The authors felt that slight trauma, which was usually held responsible for these episodes, was probably coincidental and that the origin, not only of extradural bleeding but also of subdural bleeding, was probably from a venous anomaly. One of their cases was due to a defect of blood coagulation due to anticoagulant therapy. In two cases spinal cord compression was severe and operation was undertaken later than would otherwise have been the case if the patient had initially been seen in a Department of Medical or Surgical Neurology.

REGULATION OF SPINAL BLOUD FLOW UNDER PHYSIOLOGICAL AND PATHOLOGICAL CONDITIONS

H. PAlleske, H.-D. HerRmanN, and F. LOEW (Homburg/ Saar) investigated the regulation of spinal cord blood flow in $\mathbf{4 8}$ dwarf pigs by applying a heat clearance probe to the lumbar cord at laminectomy. Simultaneously a similar probe was placed on the surface of the brain to correlate the two readings. The aorta was also exposed so that the blood supply to the lower part of the body could be occluded when required. They came to the conclusion that the regulation of the blood flow of the spinal cord was very much the same as in the brain and that the different reaction to vascular compression could be attributed to differences in the vascular pattern in the spinal cord.

This paper was then discussed by $\mathbf{K}$. Piscol (Heidelberg), R. Wüllenweber and F. K. Schröder (Bonn).

\section{MYELOPATHY IN CERVICAL SPONDYLOSIS: SURGICAL TREATMENT USING A POSTEROLATERAL APPROACH}

ANTONY JEFFERSON (Sheffield) reported a posterolateral approach for the surgical treatment of myelopathy with cervical spondylosis which had been routinely employed for the past five years. For three years, a wide laminectomy (usually from $\mathrm{C} 3$ to $\mathrm{C} 6$ inclusive) had been combined with the removal of the cartilaginous and osteophytic disc lesion. The prone cerebellar position was used. The instruments were simple. As well as the vertical incision required for the laminectomy, a transverse incision through skin and muscle was made to allow the correct trajectory of the instruments. Laminectomy was considered essential because ligamentum flavum, indenting the theca, could often be displayed on myelography. Myelograms indicating the pre- and post-operative appearances were shown, as well as a film of a recently operated patient. Among the first 50 patients, none had been worse post-operatively, $10 \%$ were unchanged, $22 \%$ had improved marginally but were still unable to work, while $68 \%$ had resumed work $(39 \%$ of this last category had a negligible disability). Analysis of a patient's clinical state at the time of operation indicated that the best results followed when the pre-operative handicaps were not gross, when hyperreflexia was not excessive, and when at least one plantar response remained flexor. After both plantar responses had become extensor, operation $-\bar{Z}$ if contemplated-should be performed as soon as con- $\mathbb{D}$ servative measures had proved themselves ineffective. It was suggested that this technique appeared both useful and safe.

\section{PROPERTIES OF SOMATOSENSORY NEURONES IN THE} HUMAN THALAMUS

A. J. MCCOMAS, P. WILSON, J. MARTIN-RODRIGUEZ, c. WALLACE, and JOHN HANKINSON (Newcastle upon Tyne) had studied the electrophysiological properties of thalamic neurones during the course of stereotaxic surgery for various movement disorders, particularly Parkinsonism, and also for intractable pain. Altogether $\frac{\Phi}{\gamma}$ 57 patients were investigated, of whom the majority were $\mathbb{\Phi}_{\Omega}$ fully conscious at the time of recording; in these patients a total of 83 tracks were made with tungsten microelectrodes.

The electrodes were directed through a posterior burr $\overrightarrow{-}$ hole towards a target in the ventralis oralis posterior $\vec{\omega}$ nucleus, $2 \mathrm{~mm}$ above the intercommisural axis at the junction of its middle and posterior thirds. The correct $\bar{\partial}$ localization of this target was greatly facilitated by a? detailed survey of neurones in the somatosensory region $\omega$ lying posteriorly (presumably $\mathbf{N}$. ventralis caudalis and $N$. ventralis intermedius). In the anteroposterior axis was possible to delineate the anterior border of thiss $\overrightarrow{1}$ somatic area, and also the interface between $N$. ventrałs oralis and the internal capsule, with considerable accuragy음 (probably to $0.5 \mathrm{~mm}$ ). In the transverse axis the evoked somatosensory potentials enabled seven degrees $\forall \mathrm{f}$ laterality to be identified. Thus, as the electrode wogs advanced anteriorly, successively more lateral tracks encountered the following sequences of topographigig. representation:

Most medial track: No evoked potentials Face (responses barely detectable) Face

Face $\rightarrow$ hand

Hand $\rightarrow$ face $\rightarrow$ hand

Hand

Most lateral track: Foot

Since the anterior border of the somatosensory nuclei occupied about $9 \mathrm{~mm}$ in the transverse axis, the recognition of seven degrees of laterality (above) conferred an accuracy of not less than $1.5 \mathrm{~mm}$ in this dimension. For most patients the best site for the centre of the lesion (6 $\mathrm{mm}$ diameter) was a point $2 \mathrm{~mm}$ beyond the anterioro border of the somatosensory region which representedo the face first and then the hand.

Of the 122 somatosensory neurones investigated to date, 86 responded to touching the skin, and 31 to move- -3 ments of joints, on the contralateral side; a further five units had high thresholds and could not be classified? satisfactorily. The joints most frequently concerned were? the metacarpo-phalangeal ones and units responding to extension were most prevalent. The receptive fields of then 'cutaneous' units varied from $0.3 \mathrm{~cm}^{2}$ to $30 \mathrm{~cm}^{2}$ in size in general, the units with the smallest fields were those 
which gave only transient discharges to maintained pressure, while those with larger fields fired repetitively. On the face the areas of skin represented most frequently were situated around the lips and on the nose; some of the larger receptive fields appeared to cross the midline. In the hand, the tips of the fingers and thumb were commonly involved. The latencies of 22 'hand' units, following electrical stimulation of the contralateral median nerve at the wrist, ranged from 13 to $19 \mathrm{msec}$. Preliminary single-unit studies had failed to demonstrate any gross changes in evoked potentials after various destructive procedures.

\section{TUBERCULOUS OSTEITIS OF THE SKULL}

JOHN MILES and BRODIE HUGHES (Birmingham) stated that tuberculosis affecting the skull had always been a relatively unusual occurrence, comprising only $1 \%$ of all tuberculous osteitis. Little had been seen of it in the Western hemisphere for perhaps 30 years. With the recent expansion of immigration to this country from areas where tuberculosis was still endemic, cranial osteitis, together with the more common forms of tuberculosis, was likely to be encountered more often.

Four cases of tuberculosis of the skull were described two of which were interesting because of neurological symptoms and signs, reputedly uncommon even when this condition was less rare. All four patients were immigrants; three presented with head pain and fluctuant swelling of the scalp. Radiographs revealed destruction of the underlying bone and they were treated by local craniectomy with appropriate antibiotics. A 15-year-old Pakistani boy developed intractable ulceration of the mouth and throat, became blind in the right eye, and lost the nasal field of his left eye. In addition he had extensive ophthalmoplegias and bulbar paresis affecting the palate, tongue, and vocal cords. Radiographs showed complete destruction of the basisphenoid with widening of the retropharyngeal space, and aspiration of the retropharyngeal abscess revealed the tuberculous nature of the infection.

Surgery for the removal of necrotic bone was still considered advisable and certainly in the treatment of massive extradural collections of pus. It was thought that chemotherapy alone would probably suffice in many cases and there was little alternative when the base of the skull was primarily involved.

Study of the literature had revealed that tuberculous osteitis was evidently a relatively benign condition, as 60 of the 87 cases operated upon up to 1933-that is, before effective chemotherapy-were said to be completely cured by surgery.

\section{CEREBELLAR HAEMANGIOBLASTOMAS}

JOHN GLEAVE (Cambridge) reviewed those cases of haemangioblastoma which had been treated at Cambridge since 1963. He stressed the importance of vertebral angiography in diagnosis and in planning treatment and commented on certain problems of anaesthesia for posterior fossa surgery.

\author{
HYPERBARIC OXYGENATION: A TREATMENT IN \\ NEUROSURGERY
}

K.-H. HOLBACH, F.-K. SCHRÖDER, G. DATENÉ, and H. DOHR (Bonn) reported their experiences in the use of hyperbaric oxygen (HO) in the treatment of traumatic post-operative and cerebrovascular cases. Their object was to institute HO therapy during the acute phase of concomitant brain oedema in an attempt to diminish secondary hypoxic damage. Patients were exposed to oxygen pressures between 2 and 3 atmospheres for periods from 30 minutes to one hour. Blood gases, $\mathrm{pH}$, lactate and pyruvate concentrations were measured; ECG and EEG recordings were made and in some cases blood pressure, pCSF, and rCBF of the affected brain tissue. Fifty-two patients were studied; 23 who had suffered severe head injuries were submitted to HO therapy on 40 occasions. On $50 \%$ of these occasions some improvement clinically was noticed -in about half of these, a marked improvement. On the second day after treatment 11 patients continued to improve, five were unchanged, and seven deteriorated. Treatment of 21 patients suffering from cerebrovascular disease produced very similar results. The remaining groups of cases were too small for analysis, but in the total assessment 22 of 52 cases showed continuing improvement, while 30 remained unchanged or deteriorated. In the cerebrovascular disease group apparent clinical improvement during $\mathrm{HO}$ therapy was comparatively rare, although a greater proportion showed improvement in the EEG record.

Although the ultimate outcome in the $42 \%$ of these patients who showed improvement with $\mathrm{HO}$ was strikingly better than in those who did not, the authors attributed this to a considerable extent to the great variability of cerebral damage and complications. They also called attention to the often transitory nature of the improvement following HO therapy and stressed the importance of a continuous EEG recording to monitor treatment.

The results of their biochemical investigations indicated that during $\mathrm{HO}$ therapy there was a rise of $\mathrm{aPO}_{2}$, while $\mathrm{PCO}_{2}$ and $\mathrm{pH}$ remained within normal limits. There was also a decrease in lactate and pyruvate concentrations indicating a reduction of anaerobic in favour of aerobic metabolism with greater energy production. Cerebral blood flow studies suggested that perfusion was influenced by varying degrees of cerebral vasoconstriction under hyperbaric $\mathrm{O}_{2}$. It was suggested that, in addition to a significant elevation of $\mathrm{aPO}_{2}$, cerebral vasoconstriction might at times be a favourable factor, as when brain swelling after injury had been associated with vasodilatation. In other circumstances, it was admitted that cerebral vasoconstriction might be a harmful influence. The authors concluded that HO therapy had been shown to be useful in certain cases and was a method deserving further study.

\section{OPERATIVE ANGIOGRAPHY}

J. W. TURNER and K. W. GROSSART (Glasgow) have employed angiography in selected cases during the course of cerebrovascular surgery. A radiolucent headrest was 